\title{
Studies of Classical Radiation Emission from Plasma Wave Undulators
}

\author{
R. L. Williams, Associate Member, IEEE, C. E. Clayton, Senior Member, \\ IEEE, C. Joshi, Fellow, IEEE, and T. C. Katsouleas, Senior Member, IEEE
}

\begin{abstract}
We examine the characteristics of the classical radiation emitted by a relativistic electron beam that propagates perpendicularly through a large amplitude relativistic plasma wave. Such a study is useful for evaluating the feasibility of using relativistic plasma waves as extremely short wavelength undulators for generating short wavelength radiation. The electron trajectories in a plasma wave undulator are obtained using perturbation techniques and are then compared to numerical simulation results. The frequency spectrum and angular distribution of the spontaneous radiation emitted by a single electron and the stimulated radiation gain are obtained analytically, and are then compared to 3-D numerical simulations. The characteristics of the plasma wave undulator are compared to the ac FEL undulator and the conventional FEL.
\end{abstract}

\section{INTRODUCTION}

$\mathbf{T}$ THERE is a significant ongoing research effort focused on producing short wavelength coherent radiation having wavelengths down to the $\mathrm{X}$-ray regime using relativistic electron beams that oscillate in undulator fields. Many examples are found in the fields of free electron lasers and synchrotron radiation sources [1], [2]. It is well known that the radiated wavelength $\lambda_{r}$ can be reduced by decreasing the undulator (wiggler) wavelength $\lambda_{w}$, or by increasing the electron energy $\gamma$ in accordance with the resonance relation

$$
\lambda_{r} \propto \frac{\lambda_{w}}{2 \gamma^{2}} .
$$

The generation of coherent radiation in the $\mathrm{X}$-ray regime using undulators was accomplished long ago by using large $\gamma$ electron beams, but this was done at very large and expensive accelerator facilities. It should be possible to reach the X-ray regime using less expensive and more compact low $\gamma$ electron beam facilities by decreasing $\lambda_{w}$. In the original undulator and synchrotron radiation schemes, and in most configurations since then, magnetic field undulators were used [3]-[5]. In magnetic field undulators, the minimum $\lambda_{w}$ obtained currently

\footnotetext{
Manuscript received May 28, 1992, revised October 6, 1992. This work was supported by the DOE under Grants DE-FG03-91-ER12114 and DEFG03-92-ER40727. The work of R. L. W. was supported by the 1990 U.S Particle Accelerator School, free electron laser course; and in part by NASA under Grant NAGW-2930.

R. L. Williams is with the Department of Physics, Center for Nonlinear and Nonequilibrium Aeroscience, Florida A. \& M. University, Tallahassee, FL 32307.

C. E. Clayton and C. Joshi are with the Department of Electrical Engineering, University of California, Los Angeles, Los Angeles, CA 90024.

T. Katsouleas is with the Department of Electrical Engineering-Electrophysics, University of Southern California, Los Angeles, CA 90089-0484.

IEEE Log Number 9206346.
}

is in the range of $0.1-1.0 \mathrm{~cm}$, and is limited by the practical difficulty of placing very strong and very small permanent magnets or electromagnets close together in an alternating array. Among alternative approaches to decreasing $\lambda_{w}$ is the replacement of the magnetic undulator with an electromagnetic wave undulator or with a crystal structure undulator, and much research is underway in these directions (for example, see [6]-[11]).

A different approach that has been suggested for decreasing $\lambda_{w}$ is to replace the magnetic undulator with an electrostatic plasma wave undulator [12]-[14]. This potentially inexpensive and compact configuration is based on using a short wavelength, large amplitude relativistic plasma wave as the undulator, through which is injected a modest energy but relativistic electron beam. In this scheme, the electrons propagate either parallel to [12]-[14] or perpendicular to [14], [15] the wavefronts of the plasma wave, and emit radiation as they oscillate in the wave's alternating electrostatic field. Such large amplitude relativistic plasma waves can be excited readily by laser pulses or intense electron bunches, and are being studied theoretically, computationally, and experimentally by many researchers around the world [16].

In this paper we consider the configuration in which the electron beam is injected across the plasma wave parallel to the wavefronts. Fig. 1 shows schematically the relationships among the propagation directions of the excitation lasers, plasma wave, electron beam, and radiation in this undulator scheme. In this particular configuration, the plasma wave is shown as if it were excited by laser beams as in the beatwave excitation scheme, however, it also could have been excited by a short laser pulse (laser wakefield scheme) or by a short pulse of relativistic electrons (plasma wakefield scheme) propagating through a plasma. The wiggling of an electron as it moves across a plasma wave undulator is described qualitatively by referring to Fig. 2, in which the potential contours of the plasma wave are shown to be very wide so that only the longitudinal electrostatic fields affect the electron motion. In the figure, the plasma wave moves to the right at a phase velocity which is nearly equal to the speed of light, while the electron moves downward also at nearly the speed of light. In Fig. 2(a), the longitudinal field deflects the electron to the left toward the potential minimum. In the time taken for the plasma wave to move to the right by one-half wavelength, the electron also moves across the wave vertically down a distance of one-half wavelength. This is because both wave and electron move at approximately the same speed. Therefore, 


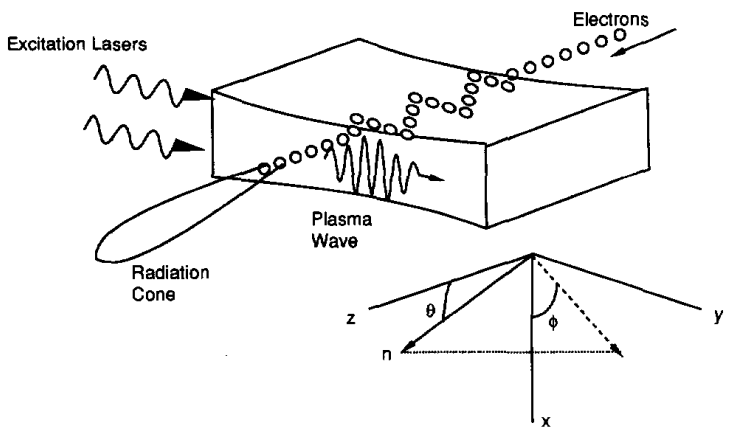

Fig. 1. Plasma wave undulator geometry, showing propagation directions of the excitation lasers, electron beam, plasma wave, and radiation.
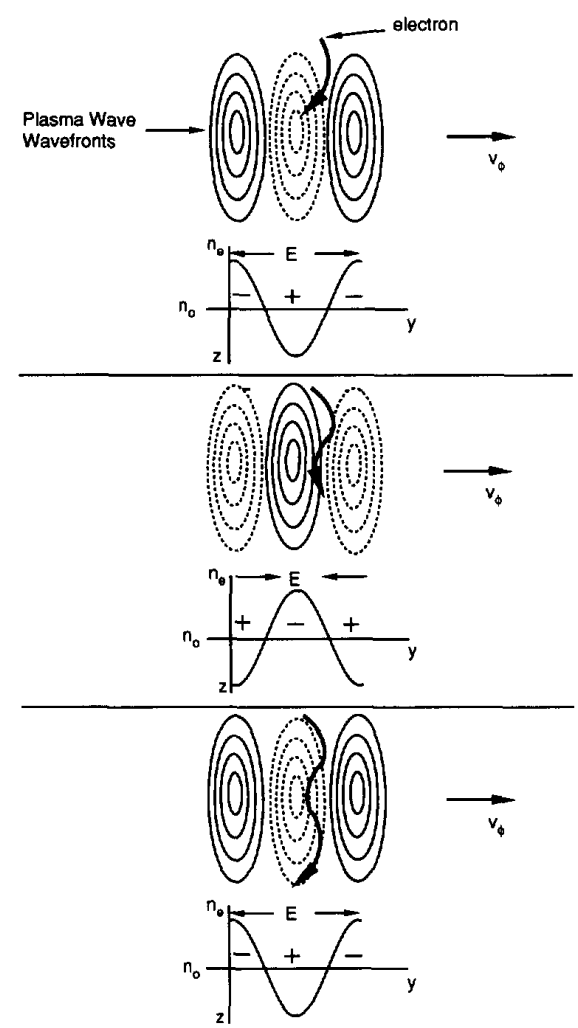

Fig. 2. Idealization of a relativistic electron's undulating trajectory in the potential contours of a relativistic plasma wave undulator.

the undulator wavelength $\lambda_{w}$ is effectively equal to the plasma wave's wavelength $\lambda_{p}$. At its new location [Fig. 2(b)], the electron is again deflected toward the potential minimum that is now to the right. This alternating or undulating motion continues as the electron passes across the plasma wave [Fig. 2(c)].

Typical physical parameters for a plasma wave undulator are: undulator wavelength, $\lambda_{w} \approx 100 \mu \mathrm{m}$; undulator strength, $a_{w} \geq 0.01 ;$ number of undulations or wiggles, $N_{w} \approx 100$; and length of undulator, $L_{w} \approx 1.0 \mathrm{~cm}$. These are typical laboratory parameters for a wide beatwave excited relativistic plasma wave. An electron beam of $20 \mathrm{MeV}(\gamma=41)$ would emit
$30 \mathrm{~nm}$ radiation in the above plasma undulator. The resonance relation (1) is used to estimate the radiation wavelength. A single 33 ps micropulse of electrons (typical of a linac output) that passes across a $1.0-\mathrm{cm}$-wide plasma undulator would emit a radiation pulse approximately $33 \mathrm{ps}$ in duration.

The maximum electrostatic fields in a relativistic plasma wave can be very large, equivalent in undulator strength to magnetic fields on the order of 1.0 MG for the case of 100 $\mu \mathrm{m}$ plasma waves. To show this we first note that $a_{w}$ for the plasma undulator can be derived from Gauss' law and is

$$
a_{w}=\frac{e E_{o}}{m_{o} c \omega_{p}}=\frac{E_{o}}{E_{\max }}=\frac{n_{1}}{n_{o}}
$$

where $E_{o}$ is the amplitude of the plasma wave electrostatic field, $E_{\max }$ is the maximum amplitude of an electrostatic field in a plasma for a given plasma density (or plasma frequency), $\omega_{p}$ is the plasma frequency, $\sqrt{4 \pi n_{o} e^{2} / m_{o}}, e$ is the electron charge, $m_{o}$ is the electron rest mass, $c$ is the speed of light, $n_{o}$ is the background plasma density, and $n_{1}$ is the perturbed plasma density. The maximum effective magnetic field $B_{\text {eff. }}$. of a plasma undulator can be estimated using

$$
B_{\text {eff. }}=\frac{2 \pi m_{o} c^{2}}{e \lambda_{p}} a_{w}=\frac{10^{4}}{\lambda_{w}(\mathrm{~cm})}=3 \times 10^{-3} \sqrt{n_{o}\left(\mathrm{~cm}^{-3}\right)}
$$

or $B_{\text {eff. }} \approx 9.5 \times 10^{5} \mathrm{G}$ for $a_{w}=1.0, \lambda_{w}=100 \mu \mathrm{m}$, and $n_{o} \approx 10^{17} \mathrm{~cm}^{-3}$. For comparison, in typical permanent magnet undulators the magnetic field is on the order of tens of $\mathrm{kG}$ and less.

As stated earlier, the relativistic plasma waves may be produced by laser beatwave excitation, laser wakefield excitation, or plasma wakefield excitation. Some typical plasma wave undulator wavelengths that can be obtained using laser plasma beatwave excitation are as follows. Using laser wavelengths of 9.6 and $10.6 \mu \mathrm{m}$ gives $\lambda_{w} \approx 97 \mu \mathrm{m}$; using 9.6 and $10.3 \mu \mathrm{m}$ laser wavelengths gives $\lambda_{w} \approx 136 \mu \mathrm{m}$; using 10.3 and 10.6 $\mu \mathrm{m}$ laser wavelengths gives $\lambda_{w} \approx 344 \mu \mathrm{m}$; and using 1.05 and $1.06 \mu \mathrm{m}$ laser wavelengths gives $\lambda_{w} \approx 102 \mu \mathrm{m}$.

In recent years, some of the problems associated with realizing a plasma undulator have been investigated theoretically and computationally. Potential problems related to the facts that the electron beam can excite instabilities and wakes in the plasma have been investigated in [12]. A limit on maximum electron beam density is imposed by the beam loading limit as discussed in [17]. Transverse electrostatic plasma wave fields should not be a problem as long as the plasma wave is much greater than a few $(\sim 5)$ plasma wave wavelengths wide [18]. Our simulation results [18] showed that electron beam quality is very important, and that the beam emittance should be less than one mm-mrad for the example parameters discussed in this paper. Methods for plasma wave excitation, calculations on the limitations of FEL action due to beam plasma interactions, and the parameters for radiation gain have been reported [12]. Some of the problems associated with building a uniform high density plasma source suitable for plasma wave undulators have been reported [19]. The problems of electron beam deflection due to the longitudinal and radial electrostatic fields in the plasma wave undulator, the effect of beam emittance, and the use of the deflected beam 
as a diagnostic of the plasma wave's electric field have also been studied through numerical calculations [18].

Several plasma-based undulators other than the scheme discussed here have also been investigated in recent years. A plasma wave undulator in which an electron beam is injected antiparallel to the wave, and in which the transverse plasma wave fields wiggle the electrons, was analyzed by Joshi et al. [12] and Fedele et al. [15]. Fedele presented a fluid theory, discussed intrinsic efficiency, emittance, self-pinching, and alternate configurations. A plasma wave undulator in which a relativistic electron beam excites the wave in a cylindrical plasma column has been investigated theoretically by Lalita $e t a l$. [20]. Latila presented an instability analysis and discussed radiation guiding. A magnetostatic plasma undulator scheme that does not involve plasma waves, but makes use of stationary rows of laser produced plasma spots (effective $\lambda_{w} \approx 100 \mu \mathrm{m}$ ) that are formed on a flat solid target has been reported, along with reports of measurements of megaGauss fields [21], [22]. Another undulator scheme has been suggested by Yan and Dawson, called the "ac Free-Electron Laser," in which an electron beam propagates through a "temporally oscillating but spatially uniform transverse electric or magnetic structure" [13]. Yan and Dawson obtained the radiation dispersion relation, expressions for the growth rate and efficiency, and discussed design criteria for an experiment. One of their configurations was to propagate an electron beam parallel to the wavefronts of a plasma wave, which was the first suggestion of the plasma wave undulator upon which this article is based. We note that in order to have a significant electric field, the plasma wave must have a phase velocity close to that of light. In such a plasma wave, the particle trajectories will be somewhat different than in the purely oscillating (ac) case. In this paper, a single electron analysis of the ac FEL undulator will be presented concurrently with the analysis of the plasma wave undulator. Finally, we note that a fluid theory analysis of the ac FEL has also been reported [23], and further work on enhancing the output power of an ac Raman FEL has been reported [24].

In the next section we describe the plasma wave equations and simulation model. Then we obtain expressions for the electron trajectories, spontaneous radiation, and stimulated radiation gain and compare these to simulation results. Many of the techniques followed herein for analyzing the plasma wave undulator, and much of the notation, are based on the free electron laser theory developed for magnetic undulators by Colson [25], [26].

\section{The Plasma Wave Equations AND THE SIMULATION MODEL}

The relativistic electrostatic plasma wave is assumed to be infinite in length, finite in width, and moving in the $+y$ direction in this model. The relativistic electrons are injected in the $+z$ direction perpendicularly through the plasma wave as shown in Fig. 1. The equation for the longitudinal electrostatic plasma wave field used in the simulations for the beat wave excited plasma wave is [27]

$$
E_{y}(x, y, z, t)=+\frac{m_{o} c \omega_{p} \alpha_{e m}}{e} e^{\left(-2 r^{2} / R^{2}\right)}
$$

$$
\times\left[\frac{1}{\alpha_{e m}} \cos \left(k_{p} y-\omega_{p} t\right)-\cos k_{p} y \sin \omega_{p} t\right]
$$

where $r^{2}=x^{2}+z^{2}, \alpha_{e m}=\left(e E_{e m} / 2 m_{o} \omega_{e m} c\right)^{2}, E_{e m}$ is the amplitude of the laser pump field and $\omega_{e m}$ is the laser pump frequency. The transverse electrostatic fields given in [15] and [28] were also used in the simulation. The electron beam model assumes point charges which interact with the electrostatic fields of the plasma wave, but which do not interact with each other through space charge forces. Radiation damping is considered negligible.

\section{ElECTRON TRAJECTORIES}

Electron trajectories in the plasma wave undulator can be calculated analytically and numerically. Approximate equations for the trajectories are obtained by substituting a simplified expression for the plasma wave field into the relativistic equation of motion and solving it by perturbation. The lowest order approximation of the electron trajectory in the plasma wave undulator is also the approximate electron trajectory in an ac FEL undulator; to next higher order, additional harmonics develop due to the nonzero $k$ of the plasma wave as well as the harmonic content of a nonlinear plasma wave. The ac FEL undulator is spatially uniform, has plasma wave wavenumber $k_{p}=0$, and oscillates at frequency $\omega_{p}$.

The plasma wave field (4) was simplified by assuming that the laser pump field or electron quiver velocity is small $\left(\alpha_{e m}\right.$ is small) and that the plasma wave is wide ( $R$ is large). Then the predominant plasma wave field component acting on the electron in the laboratory frame is

$$
E_{y}=\frac{a_{w} m_{o} c \omega_{p}}{e} \cos \left(k_{p} y-\omega_{p} t\right)=E_{o} \cos \left(k_{p} y-\omega_{p} t\right) .
$$

The trajectory is found by solving the equation of motion

$$
\frac{d \bar{v}(t)}{d t}=-\frac{e}{\gamma m_{o}}\left[\bar{E}-\frac{\bar{v}(\bar{v} \cdot \bar{E})}{c^{2}}\right]
$$

where $E=E_{y}$ and the velocity is expanded in terms of the small parameter $\epsilon$

$$
\bar{v}=\bar{v}_{o}+\epsilon \bar{v}_{1}+\epsilon^{2} \bar{v}_{2}+\cdots .
$$

First, (5) is approximated by $E_{y}=E_{o} \cos \omega_{p} t$, and the equation of motion is solved to obtain the following electron trajectory:

$\bar{r}(t)=v_{o} t \hat{z}+\frac{a_{w} c}{\gamma_{o} \omega_{p}}\left(1-\cos \omega_{p} t\right) \hat{y}+\frac{a_{w}^{2} v_{o}}{4 \gamma_{o}^{2} \omega_{p}} \sin 2 \omega_{p} t \hat{z}+\cdots$

Note that the first term is the zeroth-order drift, the second term is the first-order transverse oscillation at frequency $\omega_{p}$, and the third term is a second-order longitudinal oscillation at $2 \omega_{p}$ (the second harmonic term). The velocity $\bar{\beta}$ is found by differentiating $\bar{r}(t)$ and dividing by $c$. Equation (8) is the approximate equation for the electron trajectory in an ac FEL undulator. 


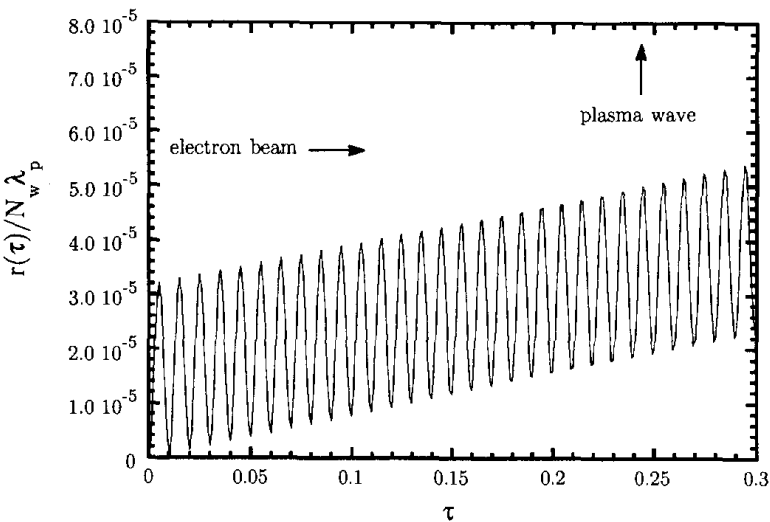

Fig. 3. Analytically calculated electron trajectory in a plasma wave undulator. The radial displacement is normalized to the length of the wiggler, $N_{w} \lambda_{p}$, and $\tau=t\left(\beta_{o} c / N_{w} \lambda_{p}\right)$.

Next, in order to obtain the approximate electron trajectory in a plasma wave undulator $\left(k_{p} \neq 0\right)$, the $y$ component of $(8)$ is substituted into (5) to obtain

$$
E_{y}=E_{o} \cos \left(\frac{k_{p} a_{w} c}{\gamma_{o} \omega_{p}}\left(1-\cos \omega_{p} t\right)-\omega_{p} t\right) .
$$

The trig terms are expanded as a series in $J_{n}(\nu)$, keeping only the lowest order terms, where $J_{n}$ is the $n$ th-order Bessel function of the first kind. Equation (9) is then substituted into the equation of motion which is again solved by perturbation. The resulting approximate equation for the electron trajectory in a plasma wave undulator is

$\bar{r}(t)=v_{o} t \hat{z}+\frac{a_{w} c J_{o}(\nu)}{\gamma_{o} \omega_{p}}\left(\cos \nu+\omega_{p} t \sin \nu-\cos \left(\nu-\omega_{p} t\right)\right) \hat{y}$

where $\nu=k_{p} a_{w} c / \gamma_{o} \omega_{p}$. In this approximation, the plasma wave field was assumed to be linear and thus was not expanded in terms of its harmonics. Using fields expanded in higher harmonics would produce additional terms in the above expressions at the respective harmonics [29]. The expressions for the trajectory and velocity obtained in this section will be used in the next section to calculate the radiation analytically.

The electron trajectory in a plasma wave undulator (10) is plotted in Fig. 3. Electrons enter the undulator at $\tau=0$ and exit at $\tau=1$. However, in order to spatially resolve the electron undulations, only the first $30 \%$ of the undulator is plotted. The undulating electrons drift in the direction that the plasma wave moves and the amount of drift increases with $a_{w}$ and decreases with $\gamma_{o}$. The analytic trajectory of an electron through the ac FEL looks similar to Fig. 3 except that there is no transverse drift.

In the numerical treatment, the original unsimplified expressions for the plasma wave fields were used, and the equation of motion was numerically integrated to obtain the electron lab frame trajectory. The 2-D lab frame trajectory calculated by simulation is shown in Fig. 4(b), which also shows a drift in the direction of the plasma wave motion, in qualitative agreement with the analytic result (Fig. 3). The Gaussian profile of the plasma wave field used in the simulation causes the drift to be

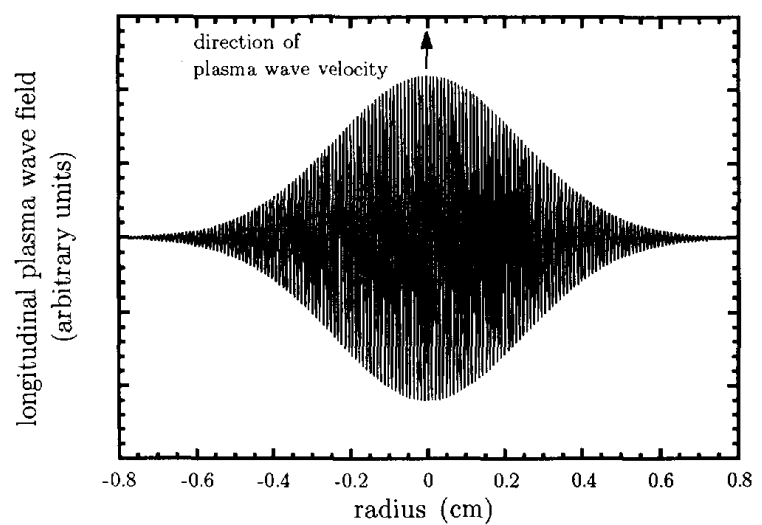

(a)

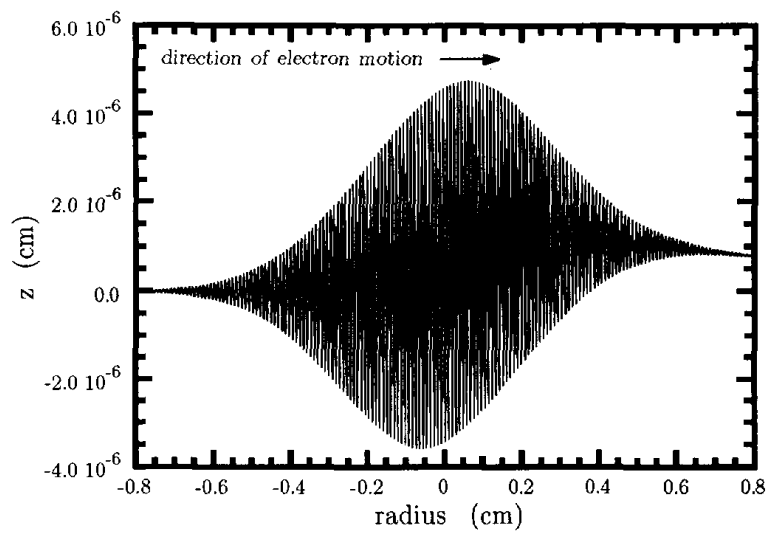

(b)

Fig. 4. (a) The longitudinal electric field seen by the electron as it traverses the plasma wave, and (b) the electron trajectory through the plasma wave; obtained by numerical simulation. The plasma wave moves upward and the electron moves to the right. Emittance $=0, \gamma_{o}=4, a_{w}=1 \%$, and $\gamma_{p}=9.7$.

more gradual at the edges of the plasma wave. The simulation also shows that there is a deflection of the electron from its initial direction of motion that results as the electron exits the plasma wave [28].

In the 2-D numerical solution, a single electron is injected into the plasma wave from a position far from the centerline of the wave $(z \approx-3 R$, where $R$ is the Gaussian width of the plasma wave). The electron's position $(x, y, z)$, normalized velocity $\left(\beta_{x}, \beta_{y}, \beta_{z}\right)$, and normalized acceleration $\left(\dot{\beta}_{x}, \dot{\beta}_{y}, \dot{\beta}_{z}\right)$ are calculated along the trajectory until the electron reaches the opposite side of the wave $(z \approx+3 R)$. The electron is injected at time equal to zero and with phase equal to zero with respect to the plasma wave (i.e., $y(0)=0$ ). The trajectory of the electron and the electrostatic field it sees are shown in Fig. 4.

In the 3-D simulation, a micropulse of thousands of electrons is injected into the plasma wave so that the front of the micropulse starts at $z \approx-3 R$. The spatial distribution of electrons in the micropulse is determined by a uniform random number generator for the direction of beam propagation $z$, and is determined by a Gaussian random number generator for the perpendicular directions $x$ and $y$. The electron micropulse has a Gaussian halfwidth of $5 \lambda_{p}$ in the $x$ and $y$ directions. 
Each electron's position, velocity, and acceleration are calculated along the trajectory until the rear of the bunch reaches the opposite side of the wave $(z \approx+3 R)$. Electron beam emittance can be included and is used to determine an initial perpendicular deflection for each electron using a Gaussian random number generator as described in [28].

\section{SPONTANEOUS RADiaTION}

The approximate equations for the frequency spectrum and angular distribution of radiation emitted by an electron in a plasma wave undulator are found by substituting the equations for $\bar{r}$ and $\bar{\beta}$ into the well-known expression for the intensity $I$ of the radiation emitted per unit solid angle $d \Omega$ and unit frequency interval $d \omega_{r}[30]$

$$
\frac{d^{2} I}{d \Omega d \omega_{r}}=\frac{e^{2} \omega_{r}^{2}}{4 \pi^{2} c}\left|\int_{-\infty}^{+\infty} \hat{n} \times(\hat{n} \times \bar{\beta}(t)) e^{i \omega_{r}(t-\hat{n} \cdot \bar{r}(t) / c)} d t\right|^{2}
$$

where $\hat{n}=\sin \theta \cos \phi \hat{x}+\sin \theta \sin \phi \hat{y}+\cos \theta \hat{z}$, and $\theta, \phi$, and $\hat{n}$ are defined in Fig. 1 . We integrate analytically over the finite interval $|t|<N_{w} \pi / \beta_{o} \omega_{p}$ because the radiation emitted during the time the electron is outside the undulator is zero. The radiation of harmonic number $f_{i}(i=1,2,3, \cdots)$ from an electron in the plasma wave undulator is then

$\left.\frac{d^{2} I}{d \Omega d \omega_{r}}\right|_{f_{i}}=\frac{e^{2}}{4 \pi^{2} c}\left[\frac{\sin \left(\frac{\omega_{r}}{\omega_{p}}\left(1-\beta_{o} \cos \theta\right)+\Delta-f_{i}\right) \frac{N \pi}{\beta_{o}}}{\left(\frac{\omega_{r}}{\omega_{p}}\left(1-\beta_{o} \cos \theta\right)+\Delta-f_{i}\right) \omega_{p}}\right]^{2}$

$\times\left(\frac{f_{i}}{1-\beta_{o} \cos \theta}\right)^{2} \times\left(\sin ^{2} \theta P P^{*}+\frac{j}{2} \sin 2 \theta \sin \phi\left(P^{*} Q-P Q^{*}\right)\right.$

$+\left(1-\sin ^{2} \theta\right) \sin ^{2} \phi Q Q^{*}$

where

$$
\begin{aligned}
P & =2 \beta_{o}\left(A_{o}+B_{n}^{+}\right)+\frac{\alpha^{2} J_{o}^{2}(\alpha) \beta_{o}}{4} \\
& \cos 2 \alpha\left(A_{2}^{+}+B_{n+2}^{+}+B_{n-2}^{+}\right) \\
Q & =\alpha A_{1}^{-}+\alpha J_{o}^{2}(\alpha) \cos \alpha\left(B_{n+1}^{-}-B_{n-1}^{-}\right) \\
A_{o} & =J_{o}(\alpha) \sum_{m=-\infty}^{m=+\infty} J_{m}(\kappa) \delta_{(2 m, f)} \\
A_{\eta}^{ \pm} & =J_{o}(\alpha) \sum_{m=-\infty}^{m=+\infty} J_{m}(\kappa)\left(\delta_{(2 m+\eta, f)} \pm \delta_{(2 m-\eta, f)}\right) \\
B_{\eta}^{ \pm} & =\sum_{n=1}^{n=+\infty} j^{n} J_{n}(\lambda) \sum_{m=-\infty}^{m=+\infty} J_{m}(\kappa)\left(\delta_{(2 m+\eta, f)} \pm \delta_{(2 m-\eta, f)}\right) \\
\alpha & =\frac{k_{p} a_{w} c}{\gamma_{o} \omega_{p}} \\
\kappa & =\frac{a_{w}^{2} J_{o}^{2}(\alpha) \beta_{o} \omega_{r}}{\omega_{p}} \cos 2 \alpha \sin \theta \sin \phi \\
\lambda & =\frac{a_{w} J_{o}(\alpha) \beta_{o} \omega_{r}}{\omega_{p}} \cos \alpha \sin \theta \sin \phi \\
\Delta & =\frac{\alpha J_{o}(\alpha) \beta_{o} \omega_{r}}{\omega_{p}} \sin \alpha \sin \theta \sin \phi
\end{aligned}
$$

$j=\sqrt{-1}, *$ signifies complex conjugate, and $\delta$ is the Kronecker delta. Here we have neglected the harmonic content of the plasma wave undulator itself. The radiation of harmonic number $f_{i}$ emitted by an electron in the ac FEL undulator can be obtained also and it is

$$
\begin{aligned}
&\left.\frac{d^{2} I}{d \Omega d \omega_{r}}\right|_{f_{i}}= \frac{e^{2}}{4 \pi^{2} c}\left[\frac{\sin \left(\frac{\omega_{r}}{\omega_{p}}\left(1-\beta_{o} \cos \theta\right)-f_{i}\right) \frac{N \pi}{\beta_{o}}}{\left(\frac{\omega_{r}}{\omega_{p}}\left(1-\beta_{o} \cos \theta\right)-f_{i}\right) \omega_{p}}\right]^{2} \\
& \cdot\left(\frac{f_{i}}{1-\beta_{o} \cos \theta}\right)^{2} \times\left(\sin ^{2} \theta P P^{*}+\frac{j}{2} \sin 2 \theta \sin \phi\left(P^{*} Q-P Q^{*}\right)\right. \\
&\left.+\left(1-\sin ^{2} \theta\right) \sin ^{2} \phi Q Q^{*}\right)
\end{aligned}
$$

where

$$
\begin{aligned}
P & =2 \beta_{o}\left(A_{o}+B_{n}^{+}\right)+\frac{\alpha_{w}^{2} \beta_{o}}{2}\left(A_{2}^{+}+B_{n+2}^{+}+B_{n-2}^{+}\right) \\
Q & =a_{w}\left(A_{1}^{-}+B_{n+1}^{-}-B_{n-1}^{-}\right) \\
A_{o} & =J_{o}(\lambda) \sum_{m=-\infty}^{m=+\infty} J_{m}(\kappa) \delta_{(-2 m, f)} \\
A_{\eta}^{ \pm} & =J_{o}(\lambda) \sum_{m=-\infty}^{m=+\infty} J_{m}(\kappa)\left(\delta_{(-2 m+\eta, f)} \pm \delta_{(-2 m-\eta, f)}\right)
\end{aligned}
$$

$B_{\eta}^{ \pm}=\sum_{n=1}^{n=+\infty} j^{n} J_{n}(\lambda) \sum_{m=-\infty}^{m=+\infty} J_{m}(\kappa)\left(\delta_{(-2 m+\eta, f)} \pm \delta_{(-2 m-\eta, f)}\right)$

$$
\begin{aligned}
\lambda & =\frac{a_{w} \beta_{o} \omega_{r}}{\omega_{p}} \sin \theta \sin \phi \\
\kappa & =\frac{a_{w}^{2} \beta_{o} \omega_{r}}{8 \omega_{p}} \cos \theta \\
k_{p} & =0 \quad \Delta=0 .
\end{aligned}
$$

The single electron radiation intensity as a function of observation angle $\theta$ and frequency ratio $\omega_{r} / \omega_{p}$ up to the third harmonic component is shown in Fig. 5 for the plasma wave undulator and, for comparison, in Fig. 7 for the ac FEL undulator. The angle is plotted in the range $|\theta|<1 / \gamma$, the harmonics correspond to the fundamental or first harmonic $f_{1}=2 \gamma^{2}$, second harmonic, $f_{2}=4 \gamma^{2}$, and third harmonic $f_{3}=6 \gamma^{2}$; and also $\phi=\pi / 2$. The radiation intensity is plotted in units of $\left(e^{2} / 4 \pi^{2} c\right)$. The figures show that the radiation is centered about $\theta=0$ in the ac FEL undulator but it is skewed in the $\theta$ direction in the plasma wave undulator. This is due to the drift of the electrons in the direction of the phase velocity of the plasma wave. There is also a reduction in the magnitude of the radiation as the harmonic number increases for both undulators. Expressions similar to (12) were obtained by Colson for the linearly polarized and circularly polarized magnetic undulators [25], [31]. These different undulator configurations have very similar radiation characteristics due to the similarity of their line shape functions. They are all characterized by regularly spaced radiation peaks, which decrease in frequency and amplitude as the angle of observation varies away from the forward direction, $\theta=0$. The skewing of the plasma undulator's radiation is due to the $\Delta$ term in the line shape function which depends on $k_{p}$ through the $\alpha$ term. By rotating 
(a)

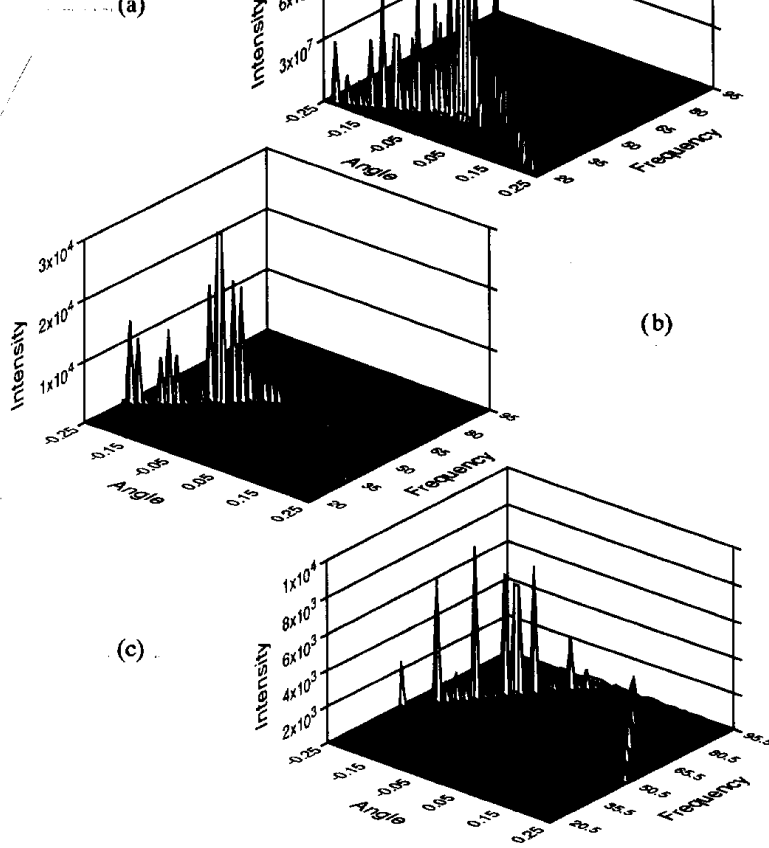

Fig. 5. Spontaneous radiation intensity emitted in the plasma wave undulato versus frequency and angle for the (a) fundamental, (b) second harmonic, and (c) third harmonic. $\gamma_{0}=4, a_{w}=0.1,(-1 / \gamma<\theta<1 / \gamma)$ $\left(\gamma^{2}<\omega_{r} / \omega_{p}<6 \gamma^{2}\right)$, and $\phi=\pi / 2$.

the $d I^{2} / d \Omega d \omega_{r}$ figures, one obtains the angular distribution $d I / d \Omega$, or the frequency spectrum $d I / d \omega_{r}$, which are shown in Figs. 6 and 8 for the fundamental harmonic of the plasma wave undulator and ac FEL, respectively. Fig. 7 shows that the ac FEL radiates the fundamental and third harmonics on axis $(\theta=0)$, but the second harmonic is radiated off axis. In general, the odd harmonics are radiated on axis and the even harmonics are radiated off axis for ac FEL as well as the linear magnetic undulator. The circularly polarized undulator radiates only the fundamental on axis and all higher harmonics are radiated off axis [25], [32].

In the simulation, the radiation intensity for the plasma wave undulator can be obtained by substituting $\bar{r}, \bar{\beta}$, and $\dot{\bar{\beta}}$ for each electron trajectory point into the well-known expression for the radiation emitted per solid angle and frequency interval given by [30]

$$
\begin{aligned}
& \frac{d^{2} I}{d \Omega d \omega_{r}}= \\
& \frac{e^{2}}{4 \pi^{2} c}\left|\int_{-\infty}^{+\infty} \frac{\hat{n} \times[(\hat{n}-\bar{\beta}(t)) \times \dot{\bar{\beta}}(t)]}{(1-\bar{\beta}(t) \cdot \hat{n})^{2}} e^{i \omega_{r}(t-\hat{n} \cdot \bar{r}(t) / c)} d t\right|^{2} .
\end{aligned}
$$

To obtain $d I / d \omega_{r}$, we numerically integrated (31) over the trajectory, squared it, and integrated over the solid angle
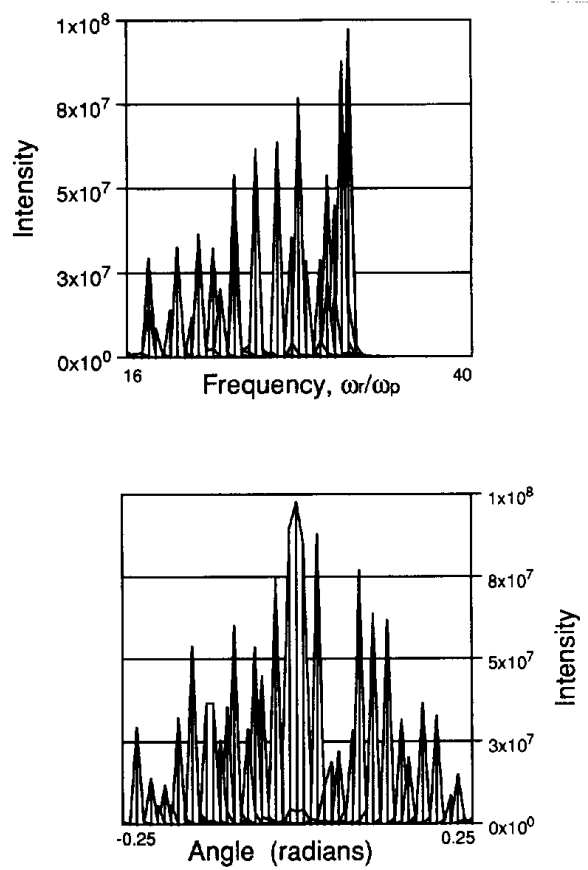

Fig. 6. Spontaneous radiation intensity spectrum and angular distribution in the plasma wave undulator for the fundamental frequency (same conditions as in Fig. 5).

(with $\phi=\pi / 2$ ). The single electron $d I / d \omega_{r}$ is shown in Fig. 9 for the fundamental frequency and zero beam emittance case, which qualitatively compares well to the approximate theoretical result for $d I / d \omega_{r}$ shown in Fig. 6(a). It was found in the simulations that as the number of electrons increased by $N$, the radiation in the peak at $\theta=0$ increased as $N^{2}$. It was also found that as the emittance was increased to values above $1 \mathrm{~mm}$-mrad, the radiation peak at $\theta=0$ decreased in magnitude, and the discrete spectral pattern became indistinguishable. This is in agreement with the beam spreading results discussed in [18].

The narrow spikes in the frequency spectrum, found analytically and by simulation, can be explained in analogy to a linear array of antennas phased so that the radiation peaks along the array axis, also called the end-fire array [33]. The electrons along the undulator radiate like antennas in line, and the radiation adds in phase along the direction of propagation. Since the length of the undulator is long compared to the radiated wavelength, the summed radiation changes rapidly as the angle of observation is changed from the forward direction. The angular width between peaks was found to be proportional to $1 / \gamma \sqrt{N_{w}}$.

The peak of the frequency spectrum $(\theta=0$ value of the fundamental) changes in accordance with the resonance relation

$$
\omega_{r}=2 \gamma_{\|}^{2} \omega_{p}=2 \gamma^{2} \omega_{p} /\left(1+a_{w}^{2}\right)
$$

as $\gamma$ and $a_{w}$ are varied. The peaks of the frequency spectra obtained from several simulations are plotted along with the theoretical resonance relation in Figs. 10(a) and (b) versus $\gamma$ 


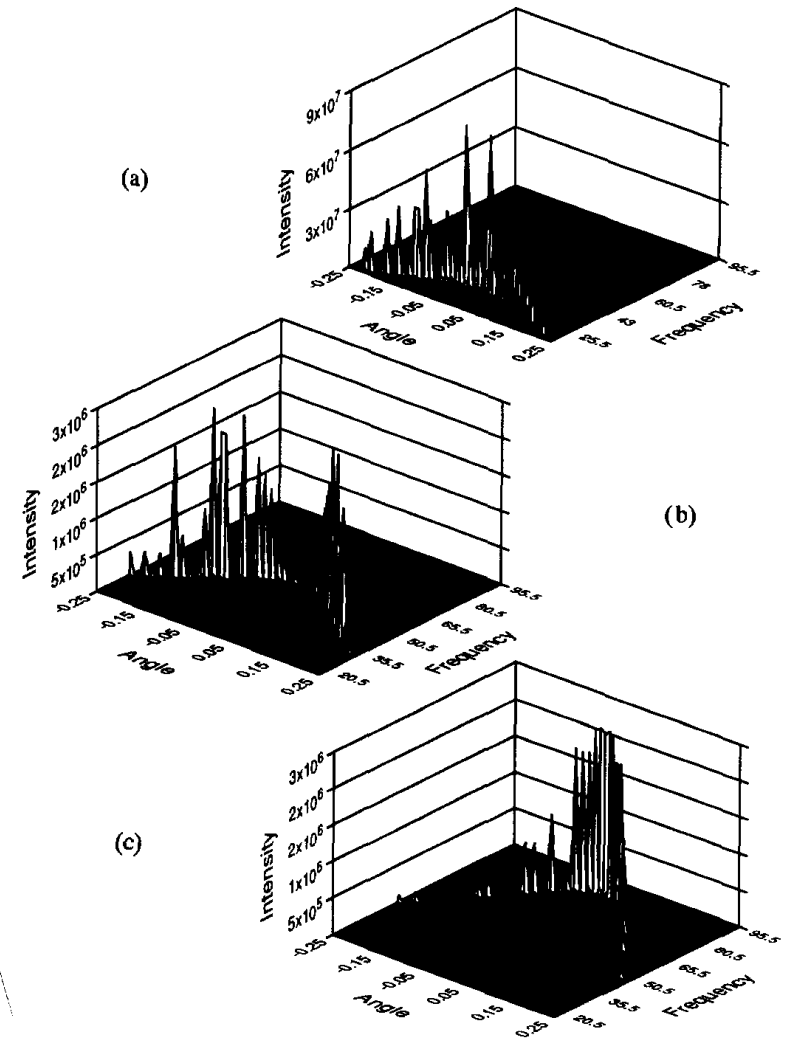

Fig. 7. Spontaneous radiation intensity emitted in the ac FEL versus frequency and angle for the (a) fundamental, (b) second harmonic, and (c) third harmonic. $\gamma_{o}=4, a_{w}=0.1,(-1 / \gamma<\theta<1 / \gamma),\left(\gamma^{2}<\omega_{r} / \omega_{p}<6 \gamma^{2}\right)$, and $\phi=\pi / 2$.

and $a_{w}$, respectively. In these figures, the solid line gives the theoretical values. The measured frequency values are given by $X$ 's when the absolute peak of the spectrum was used, and by $\circ$ 's when the frequency was measured at half way up the high-frequency slope of the spectral peak. The deviation of the $\times$ 's in Fig. 10(a) is due to the widening of the spectral peaks at $\gamma$ increases.

\section{Stimulated Radiation, Radiation GAIN, AND ElECTRON BUNCHING}

Approximate equations for the radiation gain are obtained by summing the fields of an injected plane electromagnetic (EM) wave with the plasma wave field, solving the electron equation of motion by perturbation to obtain its velocity, relating the average energy change of the electron to its average change in velocity along the undulator, and relating the change in radiation energy to the electron average energy change.

The radiation and plasma wave electric fields are oriented along the same direction so that the total fields are

$$
\bar{E}=\left(E_{o} \cos \left(k_{p} y-\omega_{p} t\right)+E_{r} \cos \left(k_{r} z-\omega_{r} t+\phi_{r}\right)\right) \tilde{y}
$$

$$
\bar{B}=E_{r} \cos \left(k_{r} z-\omega_{r} t+\phi_{r}\right) \hat{x}
$$
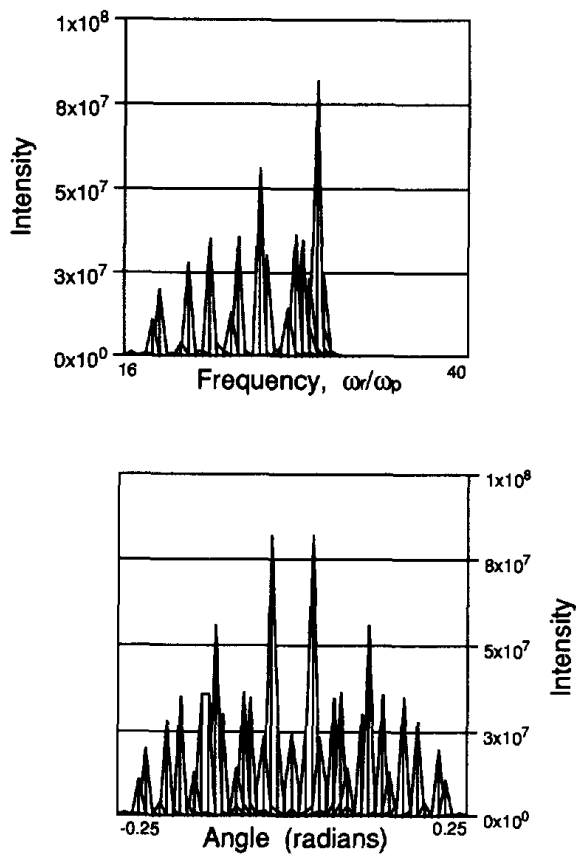

Fig. 8. Spontaneous radiation intensity spectrum and angular distribution in the ac FEL for the fundamental frequency (same conditions as in Fig. 7).

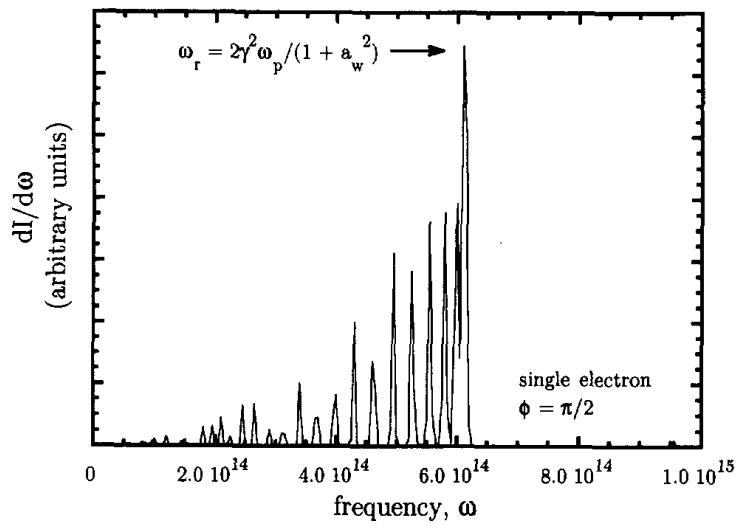

Fig. 9. Spontaneous radiation spectrum obtained by numerical simulation for the plasma wave undulator. $\gamma_{o}=4, a_{w}=1 \%$, and $\phi=\pi / 2$.

where the EM radiation has electric field strength $E_{r}$, frequency $\omega_{r}$, wave vector $k_{r}$, phase $\phi_{r}$, and magnetic field $B_{r}=E_{r}$. In this low gain approximation, $E_{r}$ is approximately constant. The frequency of the injected EM radiation field is related to the frequency of the plasma wave undulator by the resonance condition (32). An electron that is injected with energy $\gamma$ is at resonance with the radiation and does not lose or gain energy. To get radiation gain, the electrons are injected with energy slightly greater than the resonance energy, and the decrease of the average electron beam energy as it travels down the undulator results in a gain of radiation energy.

Following Colson [25], we solve the equation of motion, (6) by separating it into transverse and longitudinal components and solving for the perpendicular and parallel velocity 


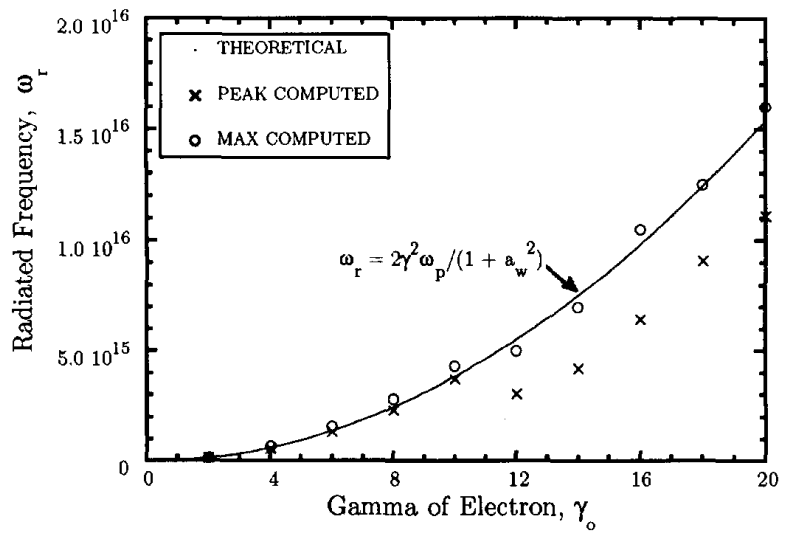

(a)

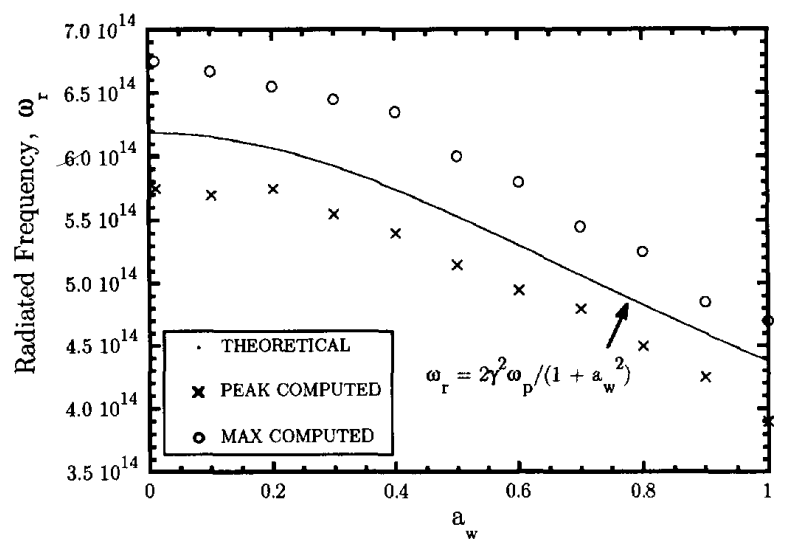

(b)

Fig. 10. Comparison of theoretical resonance condition with simulation results for the plasma wave undulator versus (a) $\gamma_{0}$ and (b) $a_{w}$.

components, $d \beta_{\perp} / d t$ and $d \beta_{\|} / d t$, which are functions of the longitudinal position $z$. The position, or phase, of the electron within a radiation wavelength is defined as

$$
\zeta(t)=\zeta_{o}+\Delta \omega t+k_{r} d z(t)
$$

where $\zeta_{o}$ is the initial phase, $\Delta \omega$ is the detuning factor, and $d z$ is the relative position of the electron in the wave. The detuning factor describes the amount that the electron's initial energy is different from the resonant energy and is

$$
\Delta \omega=\beta_{o} \omega_{p}-\omega_{r}\left(1-\beta_{o}\right)
$$

Using

$$
\frac{d \beta_{\|}}{d t}=\frac{1}{\omega_{r}} \frac{d^{2} \zeta(t)}{d t^{2}}
$$

a pendulum equation is obtained which describes the motion of the electron relative to the wave

$$
\frac{d^{2} \zeta(t)}{d t^{2}}=\Omega_{o}^{2} J_{o}(\alpha) \cos \alpha \cos \zeta(t)+a_{w}^{2} \omega_{p}^{2} \sin \left(2 \alpha-2 \omega_{p} t\right)
$$

where

$$
\alpha=\frac{k_{p} a_{w} c}{\gamma_{o} \omega_{p}}
$$

and

$$
\Omega_{o}^{2}=\frac{2 e^{2} E_{o} E_{r}}{\gamma_{o}^{2} m_{o}^{2} c^{2}}
$$

This equation can be solved by perturbation to obtain the velocity, using $\epsilon=\Omega_{o}^{2} / \Delta \omega^{2}$ as the small expansion parameter. From the change in velocity over the length of the undulator, we obtain the change in electron energy. The average final electron energy, $\langle\gamma(t)\rangle$, is found by averaging over the initial phase $\zeta_{o}$. The negative of the average change in electron energy gives the change in radiation energy, which is

$$
\begin{aligned}
& \frac{\gamma_{o}-\langle\gamma(t)\rangle}{\gamma_{o}}= \\
& \frac{\gamma_{o}^{2} \Omega_{o}^{4}}{\omega_{r} \Delta \omega^{3}} J_{o}^{2}(\alpha) \cos ^{2} \alpha\left(1-\cos \Delta \omega t-\frac{\Delta \omega t}{2} \sin \Delta \omega t\right) \\
& \quad+\frac{a_{w}^{2}}{2} J_{o}(\alpha) \cos \left(2 \alpha-2 \omega_{p} t\right)
\end{aligned}
$$

The gain, $G(t)$, is obtained by multiplying the change in radiation energy by the number of electrons, $n_{b} V$, and dividing by the EM wave energy, $2 E_{r}^{2} V / 8 \pi$, where $n_{b}$ is the beam's electron density and $V$ is the volume that contains the electron beam and EM radiation [25]. The resulting gain for the plasma wave undulator is

$$
\begin{aligned}
G(\tau)= & \frac{8 \pi e^{4} E_{o}^{2} n_{b} c}{\omega_{p} \gamma_{o} m_{o} c}\left(\frac{N_{w} \lambda_{p}}{\beta_{o} \gamma_{o} m_{o} c^{2}}\right)^{3} \frac{1}{\Delta \bar{\omega}^{3}} \\
& \times\left[J_{o}^{2}(\alpha) \cos ^{2} \alpha\left(1-\cos \Delta \bar{\omega} \tau-\frac{\Delta \bar{\omega} \tau}{2} \sin \Delta \bar{\omega} \tau\right)\right. \\
& \left.+\frac{\Delta \bar{\omega}^{3} \gamma^{4}\left(E_{o} / E_{r}\right)^{2}}{4 a_{w}^{2} \bar{\omega}_{p}^{3}} J_{o}(\alpha) \cos \left(2 \alpha-2 \bar{\omega}_{p} \tau\right)\right]
\end{aligned}
$$

where we have used the following dimensionless variables in the time varying terms:

$\tau=t \frac{\beta_{o} c}{N_{w} \lambda_{p}}, \quad \Delta \bar{\omega}=\Delta \omega \frac{N_{w} \lambda_{p}}{\beta_{o} c}, \quad$ and $\bar{\omega}_{p}=\omega_{p} \frac{N_{w} \lambda_{p}}{\beta_{o} c}$.

We note that $k_{p} c \approx \omega_{p}$ for the relativistic plasma wave and, in general, $a_{w}$ is small and $\gamma$ is large. Then the arguments of the cosine and Bessel function terms are small, thus these terms are approximately equal to one.

The pendulum and gain equations for the ac FEL undulator are obtained from (38), (41), and (42) by setting $k_{p}=$ 0 . The gain equations for the plasma wave and ac FEL undulator have terms which depend on $\bar{\omega}_{p} \tau$ and are due to the electrostatic field. The electrostatic field term also depends on the square of the ratio of the electrostatic to electromagnetic field magnitudes; and as the electromagnetic field becomes larger, the electrostatic term becomes less significant.

For comparison, we write the pendulum and gain equations for the linearly polarized magnetic wiggler [25]

$$
\frac{d^{2} \zeta(t)}{d t^{2}}=-\Omega^{2} \sin \zeta(t)
$$

and

$G(t)=\frac{4 e^{4} B_{o}^{2} n_{b} \lambda_{o}}{\left(\Delta \omega \gamma_{B} m_{o} c\right)^{3}}\left(1-\cos \Delta \omega t-\frac{\Delta \omega t}{2} \sin \Delta \omega t\right)$ 
where

$$
\begin{aligned}
\Omega^{2} & =\frac{2 e^{2} B_{o} E_{r}}{\gamma_{B}^{2} m_{o}^{2} c^{2}} \\
\gamma_{B}^{2} & =\gamma_{o}^{2}\left(1+\frac{e^{2} B_{o}^{2}}{m_{o}^{2} c^{2} \omega_{o}^{2}}\right) .
\end{aligned}
$$

$B_{o}$ is the magnet field strength, $\lambda_{o}=2 \pi / k_{o}$ is the magnetic wiggler wavelength, and $\omega_{o}=k_{o} c$.

In Fig. 11 we plot the gain versus $\tau$ (42) in units of

$$
\left(8 \pi e^{4} E_{o}^{2} n_{b} c / \omega_{p}\right)\left(N_{w} \lambda_{p} / \beta_{o} \gamma_{o} m_{o} c^{2}\right)^{3}
$$

for several values of dimensionless detuning ( $\Delta \bar{\omega}$ from 1 to 5 ) for the plasma wave undulator. At $\tau=0$, the electron enters the undulator, and exits it at $\tau=1$. For positive detuning values close to resonance ( $\Delta \bar{\omega}$ small), there is positive gain which increases as the electron travels down the undulator, as shown in the figure. As the detuning increases, the gain reaches a maximum and begins to drop near the end of the undulator. For detuning values far from resonance (i.e., $\Delta \bar{\omega}>10$ ), the gain will oscillate rapidly between positive and negative values as the electron travels along the undulator.

The case shown in Fig. 11(a) is for an electron of $\gamma_{0}=4$ in an undulator of $a_{w}=0.1$, and undulator-to-wave field ratio $E_{o} / E_{r}$ of 0.1 . The gain plots for an ac FEL undulator and a linearly polarized magnetic undulator also resemble Fig. 11(a). However, for smaller values of $a_{w}$ or larger field ratios, the electrostatic term in the gain equation becomes significant and the gain curves become as shown in Fig. 11(b) for the plasma wave and ac FEL undulators. There is a rapid oscillation superimposed upon the gain curves which is due to the electrostatic field term in the gain equation. By adjusting the terms multiplying the electrostatic term of the gain equation, this oscillation can be eliminated or increased. The physical reason we give for this oscillation is as follows. As the electron oscillates, a component of its velocity is alternately parallel and antiparallel to the electric field. The electric field can do work on the electron at a rate given by $e \bar{v} \cdot \bar{E}$ and thus can change its energy. In the magnetic undulator, the magnetic field cannot change the energy of the electrons, and therefore the gain curves are smooth.

Fig. 12 plots the gain (and absorption) versus detuning for four values of time, which represent plasma wave undulators that are shorter or longer than $\tau=1$. Positive gain occurs for small positive values of detuning, and negative gain (or absorption) occurs for small negative values of detuning. For larger values of detuning (either positive or negative), the gain is small and oscillates about zero.

The case shown in Fig. 12(a) is for an electron of $\gamma_{o}=4$ in an undulator of $a_{w}=0.1$, and undulator-to-wave field ratio of 0.1. The gain plots for an ac FEL undulator and linearly polarized magnetic undulator also resemble Fig. 12(a). The parameters of the plasma wave undulator can be chosen so that the electrostatic term becomes significant, and this is shown in Fig. 12(b) for $a_{w}=0.01$, field ratio $=3.0$, and $\gamma_{o}=4$. Fig. 12(b) shows that two of the curves oscillate about a mean value greater than zero, and two oscillate about a value less

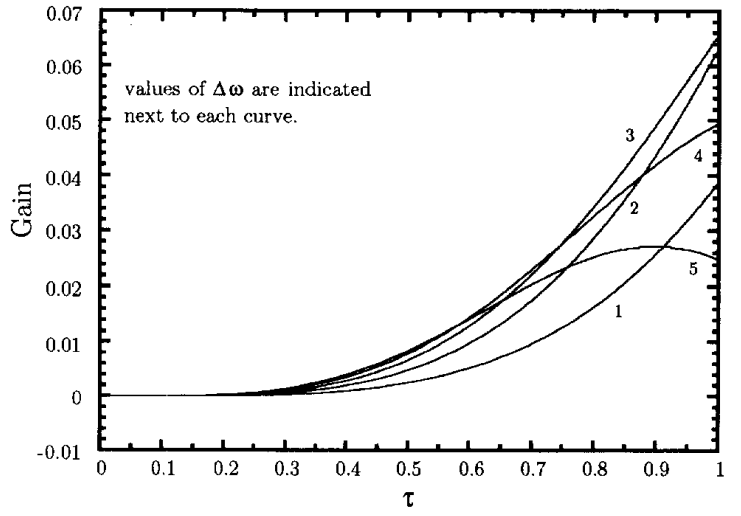

(a)

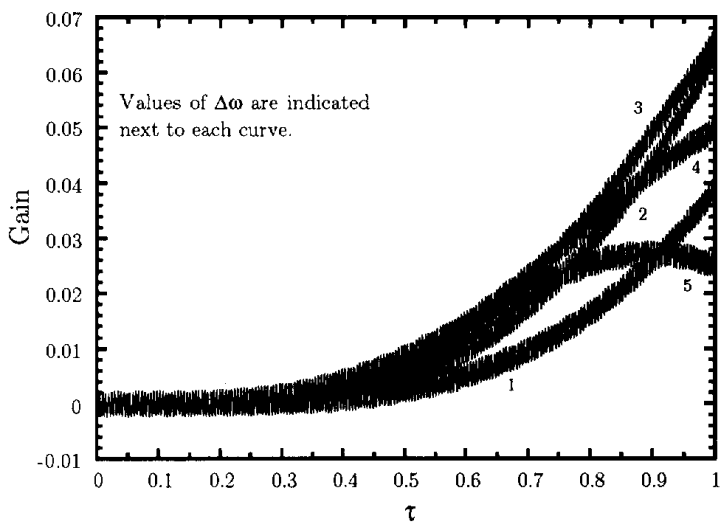

(b)

Fig. 11. Normalized stimulated radiation gain versus normalized time in the plasma wave undulator for five values of detuning, $\Delta \omega$, for (a) $E_{o} / E_{r}$ small and $a_{w}$ large, and (b) $E_{o} / E_{r}$ large, $a_{w}$ small and showing rapid gain oscillations.

than zero. If the field ratio were increased more, the separation between the two sets of curves would also increase. The parameters for Fig. 12(b) approximately correspond to those for Fig. 11(b) which has high-frequency oscillations on the gain curves. A possible explanation for the shift in the curves is that the final electron energy at the end of the undulator can be shifted higher or lower depending on whether the simulation stopped at the peak or minimum of the rapid oscillations shown in Fig. 11(b). This may explain the simulation result, however, we are not certain that this effect would occur in a physical undulator.

In the numerical simulations, a resonant EM wave was injected into the plasma wave co-parallel with a micropulse containing several thousand electrons having the same energy. Fig. 13(a) shows the spatial distribution of the electrons after emerging from the plasma wave undulator, for the case when no EM wave is present. The electron bunch is moving upward and the plasma wave is moving to the left in the figure. The perspective is looking down (in the positive $z$ direction) on the electron bunch shown in the sketch in Fig. 1. In Fig. 13(b), the EM wave is added which results in electron bunching. The separation between the large bunches in Fig. 13(b) is equal to the radiation wavelength $\lambda_{r}$. There are closer spaced 


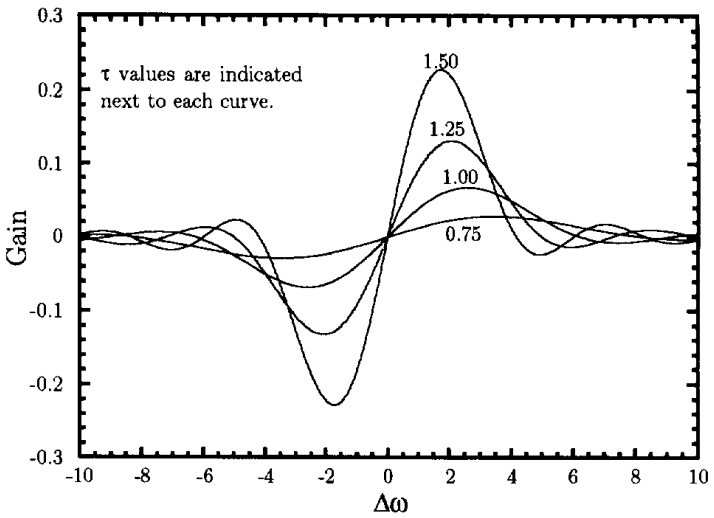

(a)

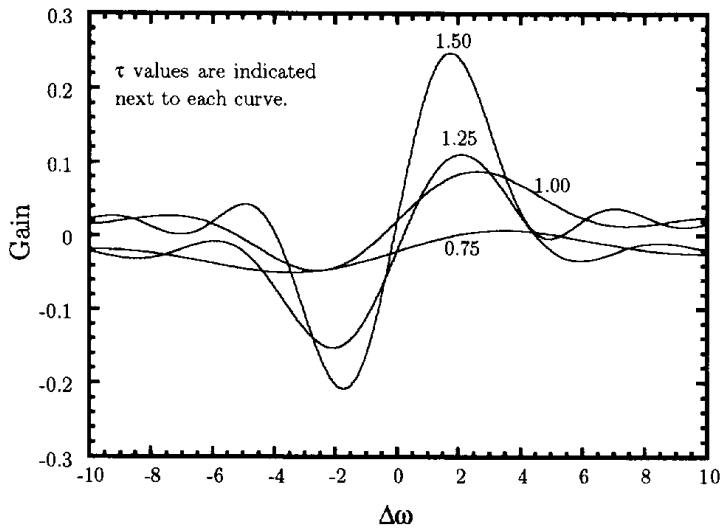

(b)

Fig. 12. Radiation gain versus detuning in the plasma wave undulator for four values of normalized time $\tau$, for (a) $E_{o} / E_{r}$ small and $a_{w}$ large, and (b) $E_{o} / E_{\mathrm{r}}$ large and $a_{w}$ small, showing gain curves offset above and below the zero gain level.

bunches within the larger electron bunches with separation approximately equal to the higher harmonic wavelengths.

In another simulation, a smaller monoenergetic group of $\mathbf{5 0}$ test electrons was injected along with an EM wave into the plasma wave undulator and allowed to drift to the end of the undulator. The final energies of the electrons were recorded and then averaged over the group of 50 . In order to simulate injection at different detuning values, each simulation run started with a different electron energy. In succesive simulation runs, the initial electron energy was swept in small increments over the range from $\gamma_{o}=3.9$ to 4.1 . The EM wave frequency was resonant with the $\gamma_{o}=4.0$ electron in each simulation run. The negative of the average energy change of the emerging electrons was plotted versus the initial energies in Fig. 14. This is the gain/absorption curve obtained by simulation that was approximated by (42) and plotted in Fig. 12. Note that the baseline of the curve is shifted above the zero gain level, and that the curve is not symmetric about the resonant energy, $\gamma_{o}=4$. If several thousand electrons had been used in the simulation (very expensive), the curve would probably have been much smoother. However, again we see that the simulation is in qualitative agreement with the results of the approximate analytic theory.

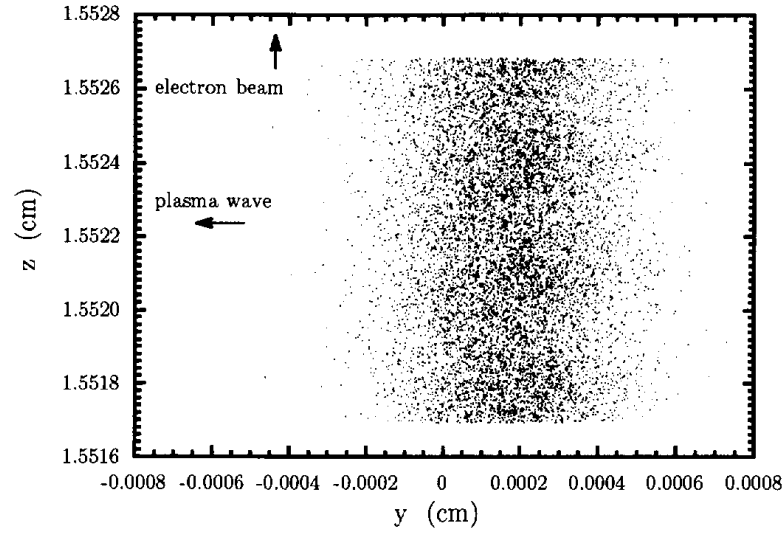

(a)

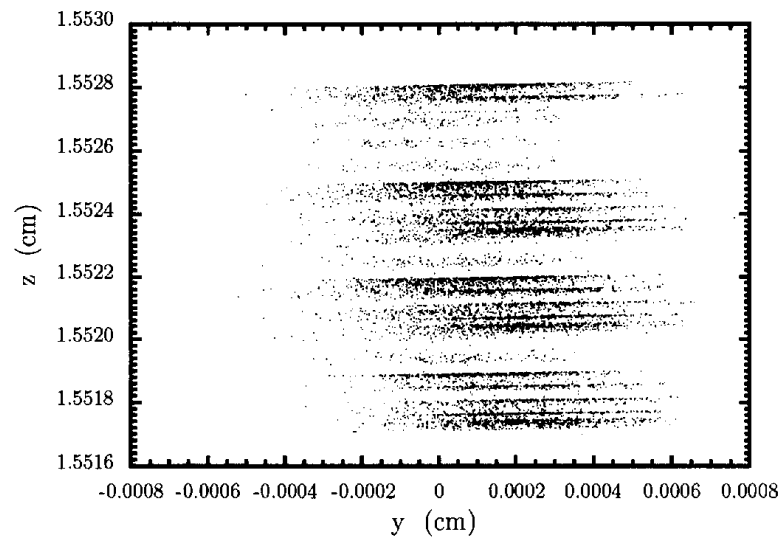

(b)

Fig. 13. Distribution of electrons emerging from the plasma wave undulator for the cases of copropagating electromagnetic wave (a) not present and (b) present, showing bunching at the fundamental and harmonic wavelengths; obtained by numerical simulation.

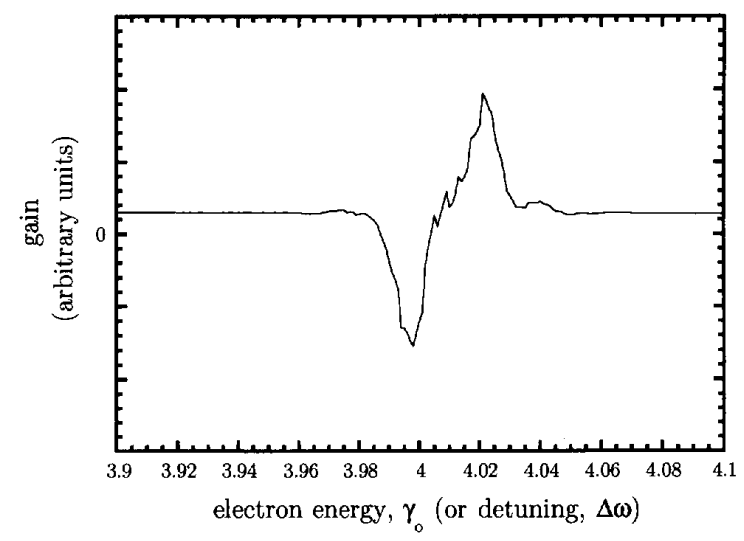

Fig. 14. Radiation gain and absorption obtained by numerical simulation versus electron injection energy $\left(\gamma_{o}\right)$, or detuning $(\Delta \omega)$. This gain curve results from combining the radiation of 50 electrons.

\section{SUMMARY}

The relativistic plasma wave undulator has the potential to be a compact and less expensive source of short wavelength radiation down to the $\mathrm{X}$-ray regime because it can be used 
with a modest energy relativistic electron beam. Approximate expressions for the electron trajectories, spontaneous radiation, and stimulated radiation gain have been obtained for the plasma wave undulator as well as for the ac FEL. The electron trajectory has a very small transverse drift in the plasma wave undulator which causes the angular distribution of the spontaneous radiation to be directed at a small angle away from the undulator centerline. The ac FEL and conventional magnetic FEL do not have this drift. The 3-D simulation results confirm our approximate analytical expressions for the trajectory, spontaneous radiation, and stimulated radiation gain. The plasma undulator also offers different harmonic content. The simulations also show that radiation gain occurs when an EM wave copropagates with the electron beam; however, a rapid modulation of the gain can also occur. Electron bunching occurs at the radiation wavelength and at harmonics when the EM wave is copropagating with the beam. The stimulated radiation gain/absorption curves resemble those of magnetic undulators, except that a shift in the gain curves above or below zero gain was observed in the simulations.

\section{ACKNOWLEDGMENT}

The authors would like to thank Dr. W. Mori for many useful discussions.

\section{REFERENCES}

[1] A. M. Sessler and D. Vaughn, "Free-electron lasers," Amer. Sci., vol. 75, pp. 34-43, Jan. 1987

[2] H. Winick, G. Brown, K. Halbach, and J. Harris, "Wigglers and undulator magnets," Phys. Today, vol. 34, pp. 50-63, May 1981. H. Winick, "Synchrotron radiation," Sci. Amer., vol. 257, no. 5, p. 88, Nov. 1987.

[3] H. Motz, "Applications of the radiation from fast electron beams," $J$. Appl. Phys., vol. 22, pp. 527-535, May 1951.

[4] J. M. J. Madey, "Stimulated emission of Bremsstrahlung in a periodic magnetic field," J. Appl. Phys., vol. 42, pp. 1906-1913, Apr. 1971

[5] C. W. Roberson and P. Sprangle, "A review of free-electron lasers," Phys. Fluids, vol. B1, pp. 3-42, Jan. 1989.

[6] S. A. Bogacz, J. B. Ketterson, and G. K. Wong, "Coherent short wave radiation from a solid state free electron laser," Nucl. Instr. Meth., vol. A250, pp. 328-336, 1986 .

[7] M. Cohen, A. Gover, and S. Ruschin, "A scheme for XUV generation based on a laser-pumped FEL with an axial electric field," Nucl. Instr. Meth., vol. A304, pp. 673-678, July 1991.

[8] A. V. Zege, "Advantages of the 'kinetic' amplification regime in a free electron laser with optical undulator," Nucl. Instr. Meth., vol. A304, pp. 520-521, July 1991.

[9] P. Dobiasch, P. Meystre, and M. O. Scully, "Optical wiggler freeelectron X-ray laser in the 5 A region," IEEE J. Quantum Electron., vol. QE-19, pp. 1812-1820, Dec. 1983.

[10] V. A. Bazylev, "Free electron laser in the ultraviolet and X-ray regions," Nucl. Inst. Meth., vol. A304, pp. 671-672, July 1991.

[11] V. G. Baryshevsky, I. Ya. Dubovskaya, and A. V. Zege, "X-ray free electron laser in a crystal as distributed feedback oscillator," Nucl. Instr. Meth., vol. A304, pp. 421-426, July 1991.

[12] C. Joshi, T. Katsouleas, J. M. Dawson, Y. T. Yan, and J. M. Slater, "Plasma wave wigglers for free-electron lasers," IEEE J. Quantum Electron., vol. QE-23, pp. 1571-1577, Sept. 1987.

[13] Y. T. Yan and J. M. Dawson, "ac free-electron laser," Phys. Rev. Lett., vol. 57, pp. 1599-1602, Sept. 1986

[14] C. Joshi, T. Katsouleas, J. M. Dawson, Y. T. Yan, and F. F. Chen, "Plasma wave wigglers for free electron lasers," in Proc. Particle Accelerator Conf., Washington, DC, IEEE Cat. 87c112387-9, 1987, p. 199

[15] R. Fedele, G. Miano, and V. G. Vaccaro, "The plasma undulator," Physica Scripta, vol. T30, pp. 192-197, 1990.

[16] R. Bingham, U. de Angelis, P. K. Shukla, and L. Stenflo, "Large amplitude waves and fields in plasmas," in Proc. Spring College Plasma Phys., Physica Scripta, vol. T30, see numerous articles therein, 1990.
[17] T. Katsouleas, S. Wilks, P. Chen, J. M. Dawson, and J. J. Su, "Beam loading in plasma accelerators," Part. Accel., vol. 22, pp. 81-99, 1987.

18] R. L. Williams, C. E. Clayton, C. Joshi, and T. Katsouleas, "Motion of relativistic electrons through transverse relativistic plasma waves," Rev. Sci. Instrum., vol. 61, no. 10, pp. 3037-3039, Oct. 1990.

[19] R. L. Williams, C. E. Clayton, C. E. Joshi, T. Katsouleas, W. B. Mori, and J. Slater, "Theory and experiments on the generation of spontaneous emission using a plasma wave undulator: A progress report," SPIE Proc. Ed., Free-Electron Lasers Appl., Series P, D. Prosnitz, Ed., vol. 1227, pp. $48-59,1990$.

[20] V. Lalita, K. Tripathi, and P. C. Agarwal, "Radiation guiding in a plasma wave wiggler free-electron laser," IEEE Trans. Plasma Sci., vol. 19, pp. 9-11, Feb. 1991

[21] A. Loeb and S. Eliezer, "Free-electron laser and laser electron acceleration based on the megagauss magnetic fields in laser-produced plasmas," Phys. Rev. Lett., vol. 56, pp. 2252-2255, May 1986.

[22] J. A. Stamper, E. A. McLean, and B. H. Ripin, "Studies of spontaneous magnetic fields in laser-produced plasmas by Faraday rotation," Phys. Rev. Lett., vol. 40, pp. 1177-1181, May 1978.

[23] T. H. Chung and J. H Lee, "Analysis of the ac free electron laser," IEEE Trans. Plasma Sci., vol. 18, pp. 651-657, June 1990.

[24] Y. T. Yan, "Output power enhancement of ac Raman free electron laser," Nucl. Instr. Meth., vol. A272, pp. 120-124, Oct. 1988.

[25] W. B. Colson, "Free electron laser theory," Ph.D. dissertation, Stanford Univ., 1977.

[26] $\{-\}\{-\}$, "One-body analysis of free electron lasers," in Physics of Quantum Electronics, vol. 5, S. Jacobs, M. Sargent, and M. Scully, Eds. New York: Addison-Wesley, 1978, Ch. 4.

[27] R. Fedele, U. de Angelis, and T. Katsouleas, "Generation of radial fields in the beat-wave accelerator for Gaussian pump profiles," Phys. Rev., vol. A33, pp. 4412-4414, June 1986.

[28] R. L. Williams, C. E. Clayton, C. Joshi, T. Katsouleas, and W. B. Mori, "Studies of relativistic wave-particle interactions in plasma-based collective accelerators," Laser Part. Beams, vol. 8, no. 3, pp. 427-449, 1990.

[29] W. B. Mori, "Theory and simulations on beat wave excitation of relativistic plasma waves," Ph.D. dissertation, Univ. Calif., Los Angeles, 1987.

[30] J. D. Jackson, Classical Electrodynamics, 2nd ed. New York: Wiley, 1975, pp. 654-700.

[31] W. B. Colson, "The nonlinear wave equation for higher harmonics in free-electron lasers," IEEE J. Quantum Electron., vol. QE-17, pp. 1417-1427, Aug. 1981.

[32] W. B. Colson, G. Dattoli, and F. Ciocci, "Angular-gain spectrum for free-electron lasers," Phys. Rev., vol. A31, p. 828, 1985.

[33] S. Ramo, J. R. Whinnery, and T. Van Duzer, Fields and Waves in Communications Electronics. New York: Wiley, 1965, Ch. 12.23.

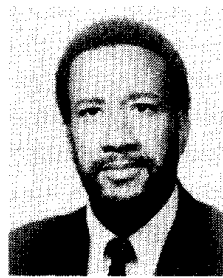

Ronald L. Williams (A'91) received the B.S. degree in engineering science from Florida State University. He received the M.S. degree in electrical engineering from Cornell University and the M.S. and Ph.D. degrees in physics from UCLA.

He was a member of the technical staff at Hughes Research Laboratory before he returned to UCLA to complete his Ph.D. His thesis was awarded the 1992 Distinguished Dissertation Prize by the NSBP. Currently, he is an Associate Professor at Florida A\&M.

C. E. Clayton (M'88-SM'92) photograph and biography not available at the time of publication.

C. Joshi, photograph and biography not available at the time of publication.

Thomas C. Katsouleas (M'88-SM'91) for a photograph and biography, please see page 52 of this issue. 